\title{
Circadian Rhythms in Isolated Brain Regions
}

\author{
Michikazu Abe, ${ }^{*}$ Erik D. Herzog,,$^{1 *}$ Shin Yamazaki, ${ }^{1}$ Marty Straume, ${ }^{1}$ Hajime Tei, ${ }^{2}$ Yoshiyuki Sakaki, ${ }^{2}$ \\ Michael Menaker, ${ }^{1}$ and Gene D. Block ${ }^{1}$ \\ ${ }_{1}^{1}$ National Science Foundation Center for Biological Timing and Department of Biology, University of Virginia, \\ Charlottesville, Virginia 22904, and ${ }^{2 H u m a n}$ Genome Center, Institute of Medical Science, University of Tokyo, Tokyo \\ 108-8639, Japan
}

\begin{abstract}
The suprachiasmatic nucleus (SCN) of the mammalian hypothalamus has been referred to as the master circadian pacemaker that drives daily rhythms in behavior and physiology. There is, however, evidence for extra-SCN circadian oscillators. Neural tissues cultured from rats carrying the Per-luciferase transgene were used to monitor the intrinsic Per1 expression patterns in different brain areas and their response to changes in the light cycle. Although many Per-expressing brain areas were arrhythmic in culture, 14 of the 27 areas examined were rhythmic. The pineal and pituitary glands both expressed rhythms that persisted for $>3 \mathrm{~d}$ in vitro, with peak expression during the subjective night. Nuclei in the olfactory bulb and the ventral hypothalamus expressed rhythmicity with peak expres-
\end{abstract}

The suprachiasmatic nucleus (SCN) is a dominant circadian pacemaker in mammals (Klein et al., 1991). In vivo, the SCN is necessary for most daily rhythms in behavior and physiology. Critically, SCN transplants restore behavioral circadian rhythms in SCN-lesioned animals with the period of the donor (Ralph et al., 1990). In vivo, various brain regions, including the SCN, substantia nigra (SN), nucleus accumbens (NA), and ventromedial hypothalamus, exhibit circadian changes of electrical activity, with the SCN peaking during the day and the others at night (Inouye and Kawamura, 1982; Inouye, 1983; Meijer et al., 1998; Yamazaki et al., 1998). However, when isolated from the SCN in vivo, either by ablating the SCN or by encircling it with a knife cut, the periodicity in extra-SCN regions is abolished (Inouye and Kawamura, 1979). These results suggest that a central pacemaker within the SCN is responsible for driving near $24 \mathrm{hr}$ rhythmicity in other regions of the brain.

The canonical view of a single pacemaker driving many circadian rhythms in mammals has been challenged recently with evidence that other tissues can generate circadian periodicities. Tosini and Menaker (1996) demonstrated that the isolated rodent

Received Aug. 22, 2001; revised Oct. 12, 2001; accepted Oct. 16, 2001.

This work was supported by National Science Foundation Science and Technology Center for Biological Timing Grant IBN 89-20162 and National Institutes of Health Grant RO1MH62517. We are grateful to Tom Breeden for programming assistance, Mika Shima for technical assistance, and Drs. Peter Brunjes and Brian Fiske for their suggestions on culturing olfactory bulb.

*M.A. and E.D.H. contributed equally to this work.

Correspondence should be addressed to Gene Block, Gilmer Hall, Department of Biology, University of Virginia, Charlottesville, VA 22904. E-mail: gdb@virginia.edu.

M. Abe's present address: Research Laboratory I, Mitsubishi Pharma Corp., Yokohama 227-0033, Japan.

E. D. Herzog's present address: Department of Biology, Washington University, St. Louis, MO 63130.

Copyright $\odot 2001$ Society for Neuroscience $0270-6474 / 01 / 210001-07 \$ 15.00 / 0$ sion at night, whereas other brain areas were either weakly rhythmic and peaked at night, or arrhythmic. After a $6 \mathrm{hr}$ advance or delay in the light cycle, the pineal, paraventricular nucleus of the hypothalamus, and arcuate nucleus each adjusted the phase of their rhythmicity with different kinetics. Together, these results indicate that the brain contains multiple, damped circadian oscillators outside the SCN. The phasing of these oscillators to one another may play a critical role in coordinating brain activity and its adjustment to changes in the light cycle.

Key words: suprachiasmatic nucleus; pineal; pituitary; olfactory bulb; arcuate nucleus; Per; luciferase; entrainment; jet lag retina exhibits a circadian rhythm in melatonin release. Furthermore, the identification of "clock genes" and the ability to measure their activity in cultured cells has led to the discovery that many non-neural tissues exhibit circadian modulations in molecular expression (Balsalobre et al., 1998, 2000; Yamazaki et al., 2000; Stokkan et al., 2001). The presence of widespread rhythmicity in mammals raises the issue of whether brain regions outside of the SCN may be capable of generating circadian rhythms. The fact that, after SCN ablation, rats show daily activity that anticipates the time of regular feeding, even in constant conditions (i.e., without food or light cycles), has led to a search for a food-entrainable, circadian oscillator (Davidson et al., 2000). In addition, although SCN transplants restore rhythmicity in SCN-lesioned hamsters, the restored period can be affected by the previous period of the host (Matsumoto et al., 1996). Whether the brain is organized around a master pacemaker driving a set of intrinsically oscillating or arrhythmic tissues has major implications in understanding the mechanistic basis of circadian properties, such as entrainment by light cycles, history-dependent after-effects, and seasonal photoperiodic regulation of behavior. Furthermore, little is known about whether circadian modulation of brain function is ubiquitous or limited to specific neural systems.

The current study takes advantage of a transgenic, Per1-luc rat model, in which it is possible to measure continuously the expression of the Period1 gene in isolated tissues. The Perl gene plays a role in the molecular mechanism underlying circadian rhythmicity in mammals (Bae et al., 2001; Zheng et al., 2001). We sought to determine the intrinsic rhythmic properties of brain regions that differ in their developmental origins or levels of Per1 expression. With the finding that some brain areas express circadian rhythmicity in vitro, we sought to characterize the resetting ki- 
netics of this rhythmicity after a shift in the animal's light schedule.

\section{MATERIALS AND METHODS}

Animals. We assessed intrinsic rhythmicity and the effects of changes in the light cycle on rhythmicity in the brain by using transgenic rats expressing the firefly luciferase gene under the control of the mouse Per1 promoter. Details on the generation of these animals and the methods have been published previously (Hida et al., 2000; Yamazaki et al., 2000; Stokkan et al., 2001). Young heterozygous or homozygous Per1-luc transgenic rats (29-43 d old) of both sexes were maintained in the animal facilities at the University of Virginia and Washington University under a $12 \mathrm{hr}$ light/dark cycle (12:12 LD). In phase-shifting experiments, the 6 $\mathrm{hr}$ advance in the light schedule was accomplished by advancing the time of lights-on, leading to one short, $6 \mathrm{hr}$ night. The $6 \mathrm{hr}$ delay was accomplished by delaying the time of lights-off, resulting in one long, 18 hr day. Rats were decapitated $1 \mathrm{hr}$ before dusk after their first, third, sixth, or 13th complete light cycle so that explanted tissues were placed into the recording incubator at the time of subjective light offset [defined as Zeitgeber time 12 (ZT 12)]. The phase of the first peak of bioluminescence in vitro was measured relative to ZT 0 (the time of light onset in the animal colony). All procedures were approved by the relevant Animal Care and Use Committees and conformed to National Institutes of Health guidelines.

Real-time Per-luc measurement. Approximately $1 \mathrm{hr}$ before dusk, rats were anesthetized with $\mathrm{CO}_{2}$ and decapitated, and their brains were rapidly removed. The pineal (Pin) and pituitary (Pit) were excised from the brain and placed in cold HBSS (catalog \#14060-057; Life Technologies, Gaithersburg, MD). Coronal sections of the brain, $400-\mu \mathrm{m}$-thick, made with a vibratome, were transferred to cold HBSS. Brain regions were identified (Paxinos and Watson, 1998) under a dissecting microscope and isolated as square tissues $\sim 1.5 \mathrm{~mm}$ across with a pair of scalpels. Cultures of SCN, retrochiasmatic nucleus $(\mathrm{RCH})$, median eminence (ME), and arcuate nucleus (AN) included both left and right nuclei, whereas all others were unilateral explants. Each culture consisted of an explant from one animal. Tissues were placed on Millicell membranes (catalog \#PICM030-50; pore size, $0.45 \mu \mathrm{m}$; Millipore, Bedford, MA) with $1.2 \mathrm{ml}$ of DMEM (Life Technologies catalog \#13000-021) supplemented with $10 \mathrm{~mm}$ HEPES, $100 \mathrm{U} / \mathrm{ml}$ penicillin, $100 \mu \mathrm{g} / \mathrm{ml}$ streptomycin, and $0.1 \mathrm{~mm}$ beetle luciferin (catalog \#E1601; Promega, Madison, WI). These cultures were sealed in a $35 \mathrm{~mm}$ Petri dish with a coverslip and vacuum grease. Cultures were maintained at $36^{\circ} \mathrm{C}$ in darkness, and their bioluminescence was continuously monitored with a photomultiplier tube (catalog \#HC135-11MOD; Hamamatsu, Shizouka, Japan) for at least $4 \mathrm{~d}$ as described previously (Geusz et al., 1997).

Acute measurement of Per-luc activity. To compare in vivo and in vitro rhythmicity of the Per1-luc reporter, we assayed bioluminescence from pineal glands explanted from transgenic rats (41-84 d old) at $3 \mathrm{hr}$ intervals for $24 \mathrm{hr}$. Rats housed in 12:12 LD were decapitated after $\mathrm{CO}_{2}$ anesthesia. During the night, we used an infrared viewer to view animals under far-red illumination (Kodak filter number 10; Eastman Kodak, Rochester, NY) during the surgery. The pineal glands from four animals at each time point were individually and rapidly frozen $\left(-80^{\circ} \mathrm{C}\right)$. Subsequently, pineal samples were thawed on ice and homogenized in lysis buffer containing $25 \mathrm{~mm}$ Gly-Gly, $15 \mathrm{~mm} \mathrm{MgSO}_{4}, 4 \mathrm{~mm}$ EGTA, $1 \%$ Triton X-100, and $1 \mathrm{~mm}$ DTT. The chilled homogenate $(50 \mu \mathrm{l})$ was diluted with $350 \mu \mathrm{l}$ of assay buffer containing $25 \mathrm{~mm}$ Gly-Gly, $15 \mathrm{~mm}$ $\mathrm{MgSO}_{4}, 4$ mm EGTA, $15 \mathrm{~mm} \mathrm{KPO}_{4}$, pH 7.8, 2 mm ATP, and $1 \mathrm{~mm}$ DTT. The bioluminescence reaction was initiated by adding $100 \mu \mathrm{l}$ of $0.4 \mathrm{~mm}$ luciferin. After a $10 \mathrm{sec}$ lag, light emission from each sample was integrated for $15 \mathrm{sec}$ by a luminometer (model TD-20/20; Turner Designs, Sunnyvale CA).

Data analysis. Phase, period, and amplitude were determined using a modification of published methods (Meerlo et al., 1997). First, data sets were detrended by subtracting the $24 \mathrm{hr}$ running average from the raw data. Then a $3 \mathrm{hr}$ and a $24 \mathrm{hr}$ running average were calculated from the detrended dataset. The crossings of these two smoothed lines provided the rising and falling phase markers for each cycle. The maximum differences between the smoothed curves for each cycle (i.e., the peak and the trough) were used to calculate the amplitude of each cycle. The time of the peak provided a third phase marker. We found that this method provided reliable results that allowed for the analysis of damped rhythmicity. The three phase markers provided similar results. For example, in 42 SCN cultures, period was found to be $24.5 \pm 0.7,24.3 \pm 0.8$, and $24.6 \pm 0.7 \mathrm{hr}$ based on the rising crossover, falling crossover, and maximum phase markers. The duration of the active $(\alpha)$ and inactive $(\rho)$ episodes of these rhythms were $11.6 \pm 0.6$ and $12.8 \pm 0.6 \mathrm{hr}$, respectively (mean \pm SEM).

Tissues that expressed only one circadian peak in vitro were considered arrhythmic. We quantified the rate at which circadian rhythmicity damped in terms of the number cycles with a period between 19 and 26 $\mathrm{hr}$ that reached or exceeded an amplitude of $30 \%$ of the first cycle. Arrhythmic (number of circadian cycles equals 0) and rhythmic cultures were included in the determination of damping rate. If rhythmicity had not damped to the criterion level by the end of the $5 \mathrm{~d}$ recording, the damping rate was interpolated from a linear fit to the amplitudes of the recorded cycles.

\section{RESULTS}

\section{Damped circadian rhythmicity is intrinsic to specific brain regions}

To assess intrinsic circadian rhythmicity of neural structures, we measured Per1-luc activity from 27 different brain regions in culture. SCN cultures showed sustained circadian rhythms in Per1-luc bioluminescence ( $n=42$ of 42 cultures) (Fig. 1). The first cycle of reporter gene activity peaked at $\sim 33.7 \pm 0.5 \mathrm{hr}$ (mean \pm SEM) after the animals experienced their last light onset or $9.7 \mathrm{hr}$ after the time of expected light onset. This phase, ZT 9.7, was consistent with previous reports of Per1 mRNA cycling in the SCN of rats (Yan et al., 1999; Yamazaki et al., 2000; Stokkan et al., 2001). SCN rhythmicity persisted for as long as we measured. The peak-to-trough amplitude of the first cycle always exceeded the amplitude of subsequent cycles and minimally decreased after 2-3 d in vitro. In 29 cultures monitored for $11 \mathrm{~d}$, amplitude decreased by $38.7 \pm 0.006 \%$ (mean \pm SEM) from the fifth to ninth cycles and by $2.5 \pm 0.007 \%$ from the ninth to 10 th cycle, when nearly one-half of the recorded rhythms actually showed slight increases in amplitude.

In accordance with previous reports on Per1 mRNA distributions (Shearman et al., 1997; Sun et al., 1997; Tei et al., 1997; Yan et al., 1999; Yamamoto et al., 2001), we found widespread Per1luc expression in the brain. Whereas the isolated SCN was rhythmic for as long as we measured, other brain regions either failed to exhibit rhythmicity or exhibited damped oscillations. The most robustly rhythmic regions were those associated with neuroendocrine function, exhibiting multiple circadian peaks of decreasing amplitude in culture. We report the results from brain nuclei grouped into four major regions.

\section{Diencephalon}

We assayed for autonomous rhythmicity in nine hypothalamic, one thalamic, and two paraneural structures. Among these, the pineal and pituitary glands oscillated longer and with the largest amplitude (Fig. 1, Table 1). In 10 of 10 pineal cultures and 16 of 16 pituitary cultures, rhythmicity persisted with decreasing amplitude for at least $3 \mathrm{~d}$. However, the phasing of the two glands was distinct, with Perl-luc expression reaching its maximum in the pineal at ZT $21.2 \pm 0.2 \mathrm{hr}$ (i.e., late subjective night; mean \pm SEM) and in the pituitary at ZT $14.3 \pm 0.8 \mathrm{hr}$ (early subjective night). Separation of the anterior and posterior lobes of the pituitary revealed that both portions expressed similar rhythms. In addition, rhythmicity in the pineal damped more rapidly than in the pituitary.

Within the hypothalamus, the AN exhibited the most robust oscillations (Fig. 1). AN cultures (18 of 19) showed between three and six cycles in vitro with a peak at ZT $14.3 \pm 0.7$. Other hypothalamic structures also oscillated but with fewer circadian peaks than the AN (Fig. 2). Notably, structures in the anteroventral hypothalamus [including $\mathrm{AN}, \mathrm{RCH}, \mathrm{ME}$, and supraoptic 


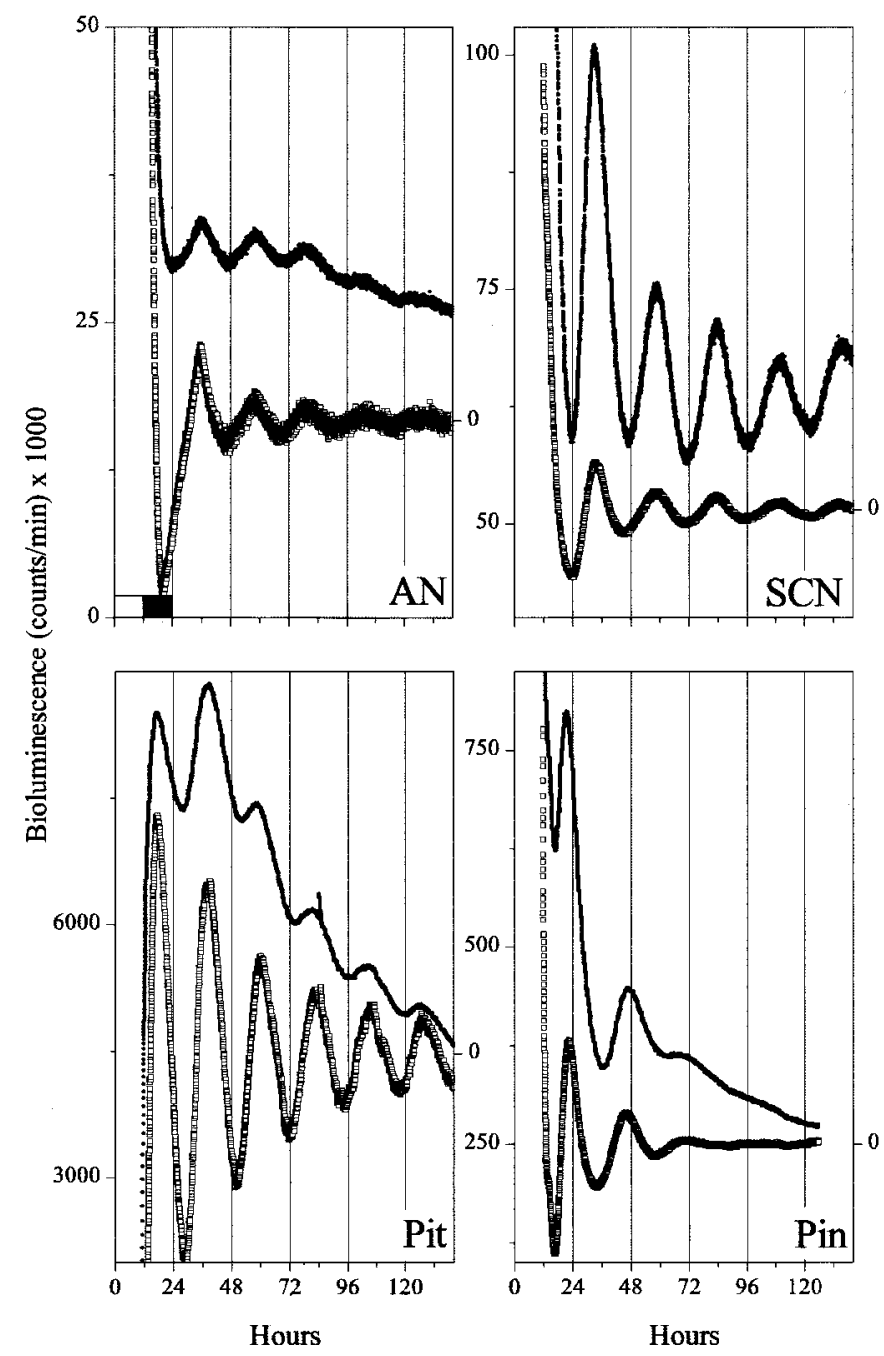

Figure 1. The SCN differs from other brain areas in the phase and amplitude of its circadian rhythmicity. Perl-luc rhythms from isolated tissues show that, whereas the SCN peaks during the subjective day, the Pin gland, Pit, and AN peak in the subjective night. In addition, circadian expression damps out in the extra-SCN regions, whereas rhythmicity persists within the cultured SCN. Tissues were explanted from Perl-luc transgenic rats $1 \mathrm{hr}$ before light offset (white and black bars in the top left plot indicate light and dark periods in the animal colony). Shown are the raw (filled circles) and detrended bioluminescence (open squares; see Materials and Methods) for representative cultures.

nucleus (SON)] all showed peak expression at approximately the time of expected dusk. In contrast, other diencephalic structures [including the lateral hypothalamus $(\mathrm{LH})$, the vascular organ of the lamina terminalis (VOLT), the paraventricular nuclei of the hypothalamus (PVN), the paraventricular nuclei of the thalamus (PVT), and the ventrolateral preoptic nucleus (VLPO)] were less likely to be rhythmic, more variable in the timing of their rhythmicity, and tended to peak later in the subjective night.

\section{Mesencephalon and rhombencephalon}

Among structures of midbrain or hindbrain origin, we assayed portions of the median (MR) and dorsal (DR) raphe, substantia nigra (SN), ventral tegmental area (VTA), and cerebellum (CE), none of which showed circadian rhythmicity (Fig. 2). There were examples of cultures for each of these tissues that showed a low-amplitude rise in bioluminescence during the second subjec- tive night in vitro, but their phases were variable and they were in the minority. This single peak may be indicative of highly damped circadian rhythmicity or a result of cell culture conditions.

\section{Telencephalon}

We sampled 10 tissues from the telencephalon. Although the piriform cortex (PC), dentate gyrus of the hippocampus (HI), and median septum (MS) all express high levels of some of the candidate clock genes, including Perl (Shearman et al., 1997; Sun et al., 1997; Tei et al., 1997; Yamamoto et al., 2001), none showed circadian rhythmicity. Other telencephalic structures that failed to express Perl-luc oscillations included the CA1, $\mathrm{CA} 2$, and $\mathrm{CA} 3$ regions of the hippocampus, caudate putamen (CP), nucleus accumbens, the posterior portion of the bed nucleus of the stria terminalis (BNSTp), and the primary motor (M1), somatosensory (S1), and auditory cortices (Au1). Cultures limited to the external pyramidal cell layer (layer III) of the somatosensory cortex (primary or barrel fields) also failed to show circadian rhythmicity.

The olfactory bulb (OB) was an exception among the telencephalic structures examined. In all cases (six of six), low-amplitude circadian rhythmicity was apparent, peaking at approximately subjective dusk. This was true in rostral and caudal sections of the bulb.

\section{Rhythmicity of the pineal in vitro reflects its in vivo phase and amplitude}

Although cell culture provides an opportunity to study properties intrinsic to isolated brain regions, it is important to know whether the properties seen in vitro represent events that normally occur in vivo. To address this, we assayed Perl-luc activity from pineal glands immediately after surgery performed at different times in the light cycle. The pineal was chosen because it can be quickly removed in its entirety and its size is comparable from animal to animal. Figure 3 shows that pineal bioluminescence increased approximately threefold from a minimum at approximately dusk to a maximum just before dawn. The phase and amplitude of this rhythm are comparable with those seen from cultured pineal glands, indicating that the isolated pineal retains its in situ phase relationship to the previous light cycle (Fig. 1).

\section{Damped rhythmicity can be restored by forskolin stimulation}

The gradual reduction in amplitude of circadian oscillations in culture could result from progressive cell death, desynchronization of multiple sustained circadian oscillators, or coincident damping of individual oscillators. It is clear that all regions taken from brain sections initially show very high levels of bioluminescence, which fall precipitously for the first few hours in vitro. This is likely attributable to the excess luciferase released into the medium from lysed cells. Brain regions incubated for $24 \mathrm{hr}$ before the addition of luciferin and initiation of recording also do not show the initial high bioluminescence levels (data not shown). We assessed health of cultures by recording their response to forskolin, a potent cAMP analog, after rhythmicity had damped out.

Circadian oscillations in the arcuate nucleus typically damped out after 2-4 d in culture. After the oscillations were severely diminished, AN cultures were treated with $10 \mu \mathrm{M}$ forskolin for 5 min (Fig. 4a). This stimulation restarted the circadian oscillation, which persisted for 3-4 cycles, with the first peak reaching a level comparable with the first cycle in vitro $(n=5)$. Medium changes did not trigger such oscillations $(n=5)$. Similarly, tissues that showed only one to three cycles after explantation, like the PVN 


\begin{tabular}{|c|c|c|c|c|c|}
\hline $\begin{array}{l}\text { Rhythmic } \\
\text { areas }\end{array}$ & $\begin{array}{l}\% \text { Rhythmic } \\
(n)\end{array}$ & $\begin{array}{l}\text { Damp rate } \\
\text { (cycles) }\end{array}$ & Phase (hr) & $\begin{array}{l}\text { Arrhythmic } \\
\text { areas }\end{array}$ & $n$ \\
\hline SCN & $100(42)$ & $>10$ & $9.7 \pm 0.5$ & $\mathrm{Au} 1$ & 2 \\
\hline Pin & $100(10)$ & $1.3 \pm 0.4$ & $21.2 \pm 0.2$ & BNSTp & 3 \\
\hline Pit & $100(16)$ & $2.9 \pm 0.3$ & $14.3 \pm 0.8$ & $\mathrm{CE}$ & 3 \\
\hline $\mathrm{RCH}$ & $100(4)$ & $1.8 \pm 0.1$ & $11.7 \pm 0.1$ & $\mathrm{CP}$ & 5 \\
\hline $\mathrm{ME}$ & $100(2)$ & $1.3 \pm 0.3$ & $11.5 \pm 1.8$ & DR & 6 \\
\hline OB & $100(6)$ & $1.6 \pm 0.4$ & $11.5 \pm 0.3$ & HI & 13 \\
\hline AN & $95(19)$ & $1.7 \pm 0.2$ & $14.3 \pm 0.7$ & M1p & 3 \\
\hline PVN & $92(12)$ & $1.0 \pm 0.0$ & $17.1 \pm 0.6$ & MR & 3 \\
\hline LH & $75(4)$ & $0.8 \pm 0.3$ & $14.4 \pm 3.4$ & MS & 7 \\
\hline VOLT & $50(8)$ & $0.8 \pm 0.5$ & $17.3 \pm 2.3$ & NA & 6 \\
\hline VLPO & $43(7)$ & $1.1 \pm 0.5$ & $18.7 \pm 1.8$ & $\mathrm{PC}$ & 10 \\
\hline PVT & $40(5)$ & $1.0 \pm 0.0$ & $22.4 \pm 5.6$ & S1 & 8 \\
\hline \multirow[t]{2}{*}{ SON } & $38(8)$ & $0.4 \pm 0.2$ & $13.0 \pm 3.1$ & $\mathrm{SN}$ & 7 \\
\hline & & & & VTA & 4 \\
\hline
\end{tabular}

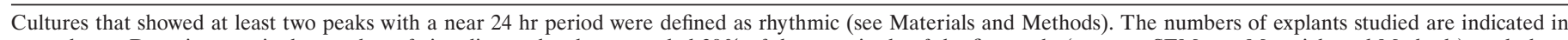

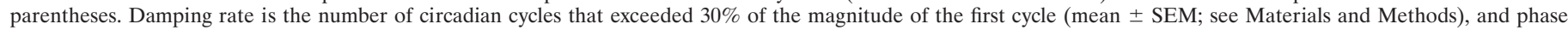
is the time of the first circadian peak relative to the animal's previous light cycle in which dawn and dusk occurred at 0 and 12 hr, respectively.

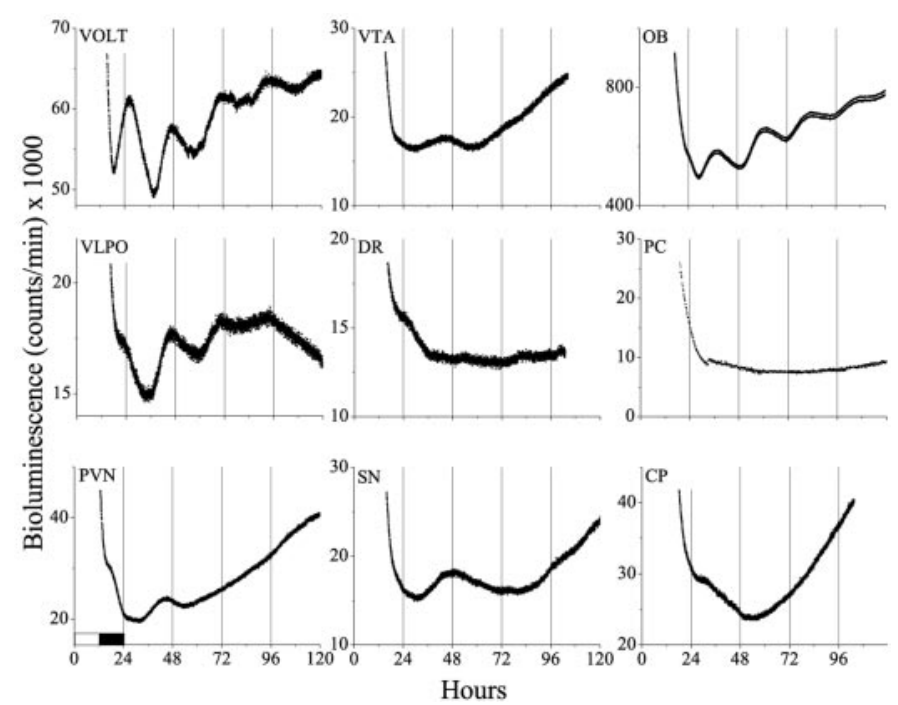

Figure 2. Circadian rhythmicity is not ubiquitous in the brain. Brain areas of diencephalic origin, such as the VOLT, VLPO, and PVN, showed damped circadian rhythmicity for one to four cycles in vitro, whereas most others did not (e.g., VTA, DR, SN, PC, and CP). The OB was the only telencephalic structure tested that showed circadian rhythmicity.

$(n=2$ of 5$), \operatorname{RCH}(n=1$ of 1$)$, Pit $(n=2$ of 2$)$, and VOLT $(n=$ 1 of 4 ), responded with a comparable number of damped circadian cycles after forskolin application. A medium change was sufficient to reinstate rhythmicity in OB cultures $(n=4$ of 4$)$. In all tissues tested, forskolin evoked a transient increase in Per1-luc activity. The treatment did not elicit circadian rhythmicity in arrhythmic tissue-types [NA, $n=4$ (Fig. $4 b$ ); CP, $n=2$; SON, $n=2$; SN, $n=3 ; \mathrm{S} 1, n=2$; and MS, $n=2$ ]. These results suggest that the potential for autonomous oscillation is different between brain regions and that damped rhythmicity does not result primarily from a gradual decline in the health of the tissue but is more likely a characteristic of the circadian oscillators in that region.

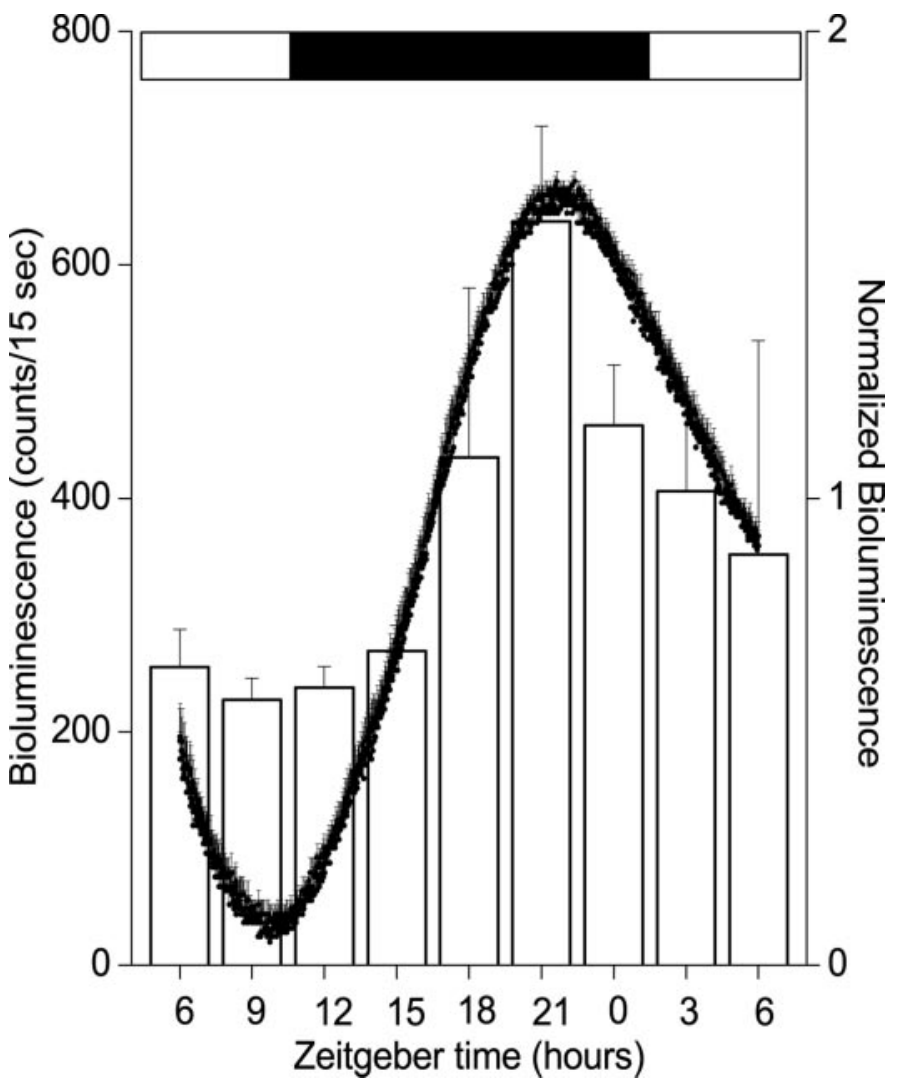

Figure 3. Pineal rhythmicity is similar in vivo and in vitro. Reporter construct activity was assayed from explanted pineal glands, either acutely at different times in the light cycle (open bars; mean \pm SEM; $n=4$ at each time point) or from cultured glands in continuous darkness ( filled circles; $n=6$ ). Bioluminescence profiles from cultured pineals were normalized to their peak emission. Acute and continuous measurements peaked $\sim 3$ $\mathrm{hr}$ before light onset. 


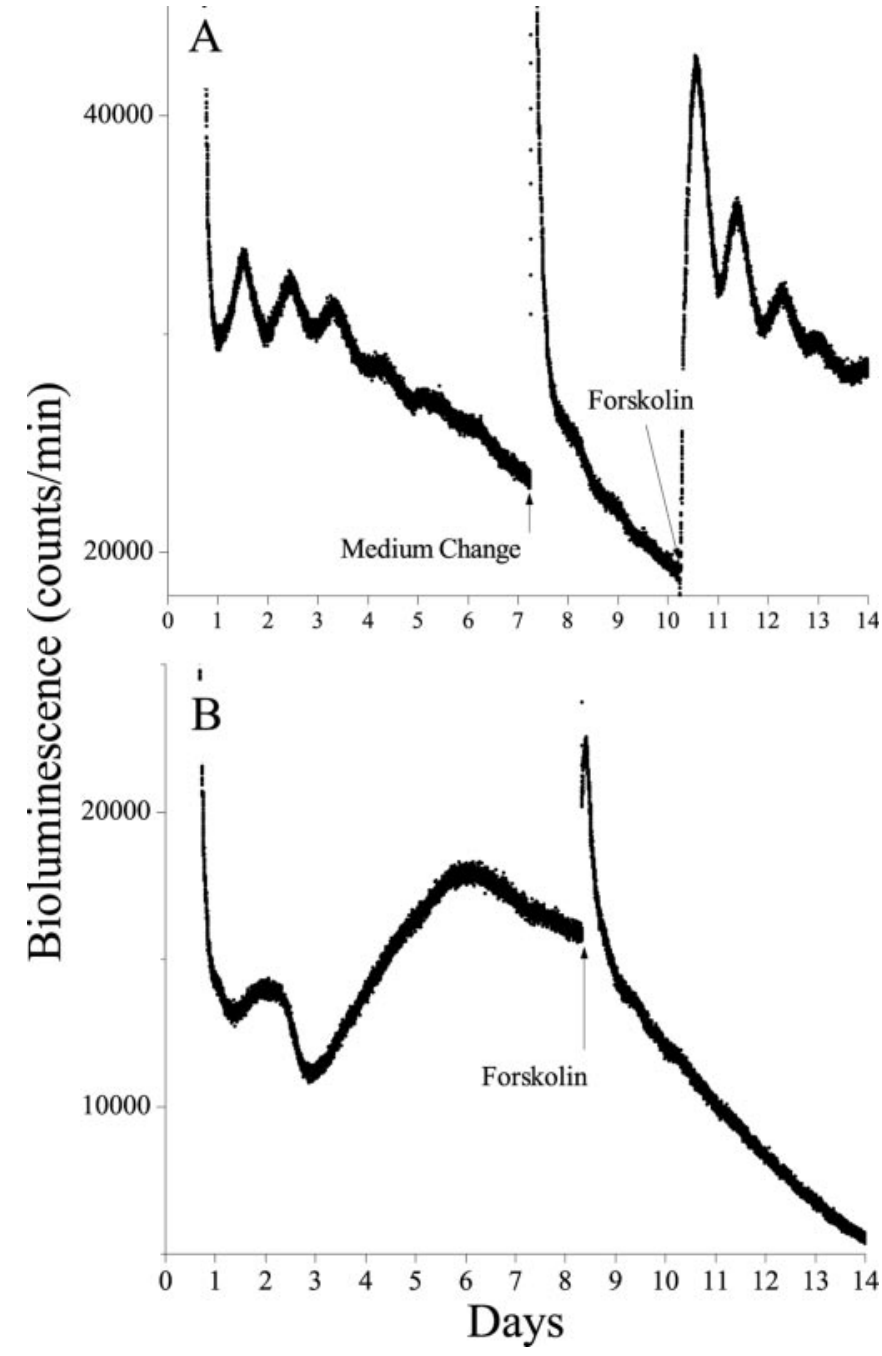

Figure 4. Damped circadian rhythmicity can be reinstated in tissues that were previously rhythmic in vitro. Although changing the medium had little effect on bioluminescence in transgenic AN cultures, exposure to forskolin $(5 \mathrm{~min}, 10 \mu \mathrm{M})$ evoked circadian oscillations that damped over the following $4 \mathrm{~d}(A)$. In contrast, rhythmicity was not seen in cultured NA, even after forskolin treatment $(B)$. These results indicate that these tissues are healthy in vitro and retain different abilities to express autonomous circadian rhythmicity.

\section{Shifts in the light schedule disrupt the normal phase relationships among brain oscillators}

We evaluated the resetting kinetics of three extra-SCN brain areas to a $6 \mathrm{hr}$ advance or delay in the light schedule. We chose the AN, PVN, and pineal because the phase of their first peak was consistent in culture. Perl-luc rats were killed one to six cycles after the phase advance or one to 13 cycles after the phase delay. Those cultures from which we could measure the phase from the first circadian peak were defined as rhythmic. When cultures were taken from rats $1 \mathrm{~d}$ after the advance in their light schedule, the AN peaked $\sim 3$ hr later than in unshifted animals (Fig. $5 a$ ). Only after the animals experienced 3-6 d in the advanced light cycle did their AN peak at the expected phase. After a delay in the light schedule, two of six AN cultures were arrhythmic, with the remaining four cultures expressing a rhythm that peaked $\sim 3 \mathrm{hr}$ before controls. When cultures were taken from animals $3 \mathrm{~d}$ after the delay in the light schedule, rhythmic explants peaked within $0.6 \mathrm{hr}$ of the phase expected after resynchronization to the light

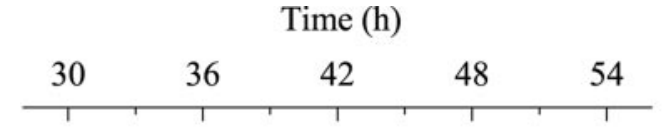

A Arcuate Nucleus

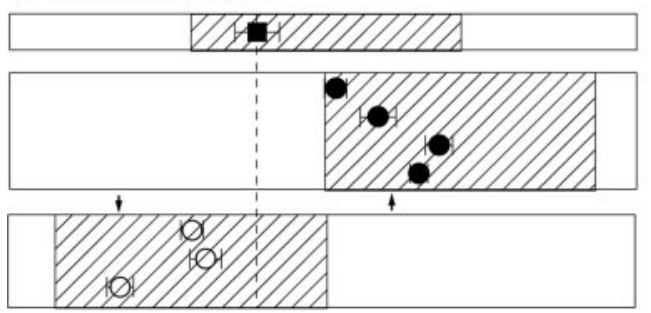

Control (10/13)

B Paraventricular Nucleus

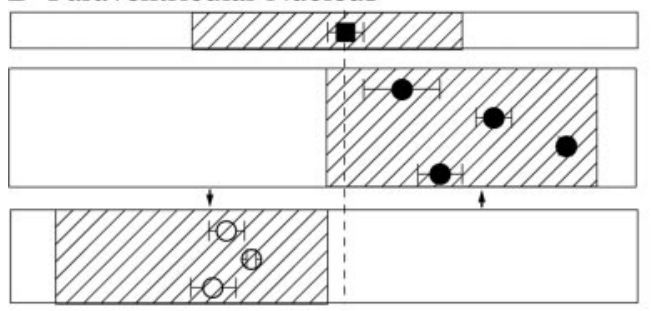

Control $(8 / 12)$

1 st $(3 / 6)$

3rd $(5 / 6)$

6th $(6 / 6)$

13 th $(6 / 6)$

1 st $(5 / 6)$

3rd $(3 / 6)$

6 th $(5 / 6)$

C Pineal

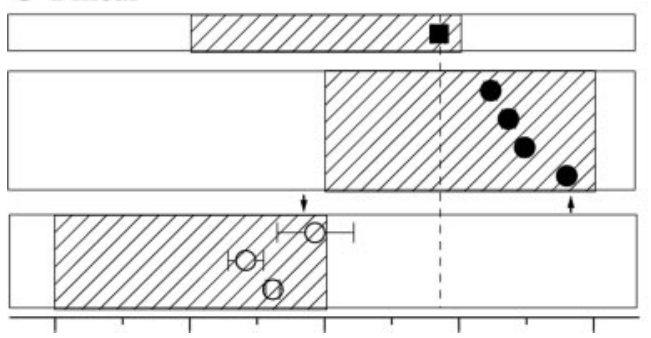

Control (6/6)

1 st $(6 / 6)$

3rd $(6 / 6)$

6th $(6 / 6)$

13 th $(4 / 4)$

1 st $(3 / 6)$

3rd $(6 / 6)$

6th $(6 / 6)$

Figure 5. Brain areas reentrain at different rates after a shift in the light schedule. Relative to tissues taken from unshifted animals ( filled squares), tissues taken from animals that experienced a $6 \mathrm{hr}$ delay (filled circles) or advance (open circles) took many days to resume their normal phase relationship to the light cycle. Plotted against time since the last light onset of the control group, rhythmicity in the arcuate nucleus $(A)$ peaked $3 \mathrm{hr}$ later than usual after $1 \mathrm{~d}$ in the delayed light cycle and appeared close to entrained after $3 \mathrm{~d}$ in the new schedule (numbers in parentheses indicate rhythmic cultures per number of cultures tested, and arrows indicate the point of complete phase shift). After a $6 \mathrm{hr}$ advance, the AN assumed its normal phase after $6 \mathrm{~d}$ in the novel light cycle. In contrast, the PVN $(B)$ and pineal $(C)$ appeared to reentrain more rapidly to the advance but took much longer to synchronize to the delayed schedule.

cycle. However, when taken from animals that remained for 6 or $13 \mathrm{~d}$ in the new schedule, the AN actually appeared to slightly overshoot the phase of control AN.

The PVN also adjusted slowly to the delayed light cycle (Fig. $5 b)$. Only three of six explants removed from animals after $1 \mathrm{~d}$ in the new light cycle showed circadian rhythmicity, peaking $3.5 \mathrm{hr}$ before the phase of controls. PVN remaining in the animal for 2 more days showed an average peak expression $\sim 0.6 \mathrm{hr}$ later than the expected phase. However, as in the case of the AN, the PVN appeared to overshoot its predicted phase relationship (by $\sim 3.8$ hr) after $6 \mathrm{~d}$ in the new schedule and then recover by the 13th day. In contrast, the PVN shifted nearly completely and stably to its appropriate phase within one cycle in the advanced light schedule.

The pineal also shifted quickly after an advance in the light cycle but slowly reentrained to a $6 \mathrm{hr}$ delay over the course of $13 \mathrm{~d}$ (Fig. 5c). Six cycles after the phase shift, the pineal had accom- 
plished only approximately one-half of the adjustment to the new light schedule. After $13 \mathrm{~d}$, the pineal appeared to have reentrained.

\section{DISCUSSION}

The forebrain, midbrain, and hindbrain form the fundamental or developmental divisions of the mammalian brain. Although the Period1 gene is widely expressed in these divisions, its level and pattern of expression depend on location. Per1 transcription is circadian in a variety of isolated tissues in vitro, but rhythmicity is neither ubiquitous nor restricted only to those that highly express Per1. For example, the hippocampus and piriform cortex, although among the areas with the highest levels of Perl, show no intrinsic rhythmicity, whereas tissues with similar (e.g., suprachiasmatic nucleus and olfactory bulb) or lower (e.g., arcuate nucleus) expression demonstrate the ability to oscillate in vitro with a near $24 \mathrm{hr}$ period for multiple cycles.

Two major differences exist among the tissues that show intrinsic rhythmicity: the strength and phase of their rhythmicity. Whereas the isolated SCN sustains daily rhythms with little reduction in amplitude for $>11 \mathrm{~d}$, rhythmicity in other mammalian tissues appears to damp out. In some structures, such as the pineal, pituitary, and arcuate nucleus, this rhythmicity persists, with decreasing amplitude, for as many as $6 \mathrm{~d}$. In others, such as the supraoptic nucleus, rhythmicity rarely shows more than one cycle in vitro. Of the 27 brain areas surveyed in this study, approximately one-half were rhythmic, and only the SCN showed peak expression during the day. Other nuclei within the anteroventral hypothalamus and the olfactory bulb reached their maximal expression several hours later, at approximately dusk. The remaining rhythmic structures (PVN, PVT, VLPO, and VOLT) peaked during the night. This is consistent with the observed diurnal electrical activity of the SCN and nocturnal activity in extra-SCN brain regions (Inouye and Kawamura, 1979; Kubota et al., 1981; Yamazaki et al., 1998).

Autonomous circadian oscillations lasting for more than two cycles in vitro have been reported recently for a number of organs, including liver, lung (Yamazaki et al., 2000; Stokkan et al., 2001), and retina (Tosini and Menaker, 1996). Immortalized fibroblast cells also show damped rhythmicity after exogenous stimulation (Balsalobre et al., 1998). Compared with these cell types, most non-SCN brain regions are either arrhythmic or only weakly rhythmic. Rhythmicity in vitro is likely to reflect in vivo properties because the phase of rhythmicity is consistent in both conditions. Furthermore, brain areas that damp show a healthy response to exogenous stimulation and damped rhythmicity is restored for several cycles. The absence or weakness of oscillations in some brain regions may allow for strong phase control by the SCN. On the other hand, more robust oscillations may provide increased lability and a wider range of phase relationships between the SCN and the target tissue. A full appreciation of temporal organization within the CNS will require a better understanding of how rhythmicity impacts the physiological functions subserved by particular brain regions.

It is not obvious why some brain regions exhibit more robust oscillations than others. It is clear that the tissue-level expression of Perl does not predict the oscillating capacity of the tissue. Developmentally, the various oscillating tissues do not share a common origin, although, in the brain, structures of diencephalic origin showed more robust rhythmicity than other areas and all peaked during the night, except the SCN. Furthermore, circadian rhythm generation is likely cell autonomous in the SCN (Welsh et al., 1995; Liu et al., 1997; Herzog et al., 1998), but it is unknown whether damped circadian rhythmicity (or arrhythmicity) is a property of single cells or the result of rhythmic cells rapidly drifting out of phase from one another. It is possible that those regions that failed to show circadian rhythmicity were adversely affected by the culture conditions. This is unlikely to be attributable to massive cell death because forskolin evoked a healthy response or to be attributable to a loss of neurons because long-term recordings from hippocampal neurons also failed to reveal circadian rhythms in firing rate (Welsh et al., 1995). Regional differences in gene expression or redox state have been proposed to affect their ability to generate circadian rhythms (Rutter et al., 2001).

Another difference among these extra-SCN circadian oscillators lies in their adjustment to changes in the light schedule. Previous research has shown that the phase of Perl expression in the SCN rapidly adjusts to large shifts in the light schedule (Albrecht et al., 1997; Yamazaki et al., 2000). We showed recently that, based on Perl activity, the lung, liver, and kidney exhibit much slower rates of reentrainment to shifted light schedules (Yamazaki et al., 2000). Assuming that mPer1 activity reflects the state of these various circadian oscillators, this result has been interpreted to suggest that internal desynchronization occurs between the central oscillator located within the SCN and a number of "peripheral" oscillators. The present results extend these findings to suggest that the brain itself undergoes internal desynchronization during changes in environmental light cycles. Internal desynchrony, which was first observed in human "bunker experiments" (Aschoff and Wever, 1976), may play a role in the malaise associated with transmeridian travel. The markedly different phase resetting kinetics among the arcuate, paraventricular nucleus, and pineal raise fundamental questions about whether some of the "circadian characteristics" of behavioral rhythms are attributable to "supra-SCN" multi-oscillator organization, i.e., whether the commonly observed transient cycles in behavior during light-induced phase advances is attributable to internal desynchronization and readjustment among multiple oscillators within the CNS.

These results provide important insights into the organization of the mammalian circadian timing system. Of the many circadian rhythms in physiology and behavior, nearly all are abolished by SCN lesions. Because SCN transplants can restore behavioral rhythms, it seems likely that the SCN impresses rhythmicity on brain areas responsible for rhythmic behaviors. However, there are two conceptually distinct mechanisms by which the SCN may coordinate rhythmic behaviors. The SCN may act as the sole pacemaker that drives circadian rhythmicity in otherwise arrhythmic brain regions. Alternatively, the SCN may serve as a primary circadian pacemaker that entrains damped and/or weakly coupled oscillators resident in other regions of the nervous system. Studies using in vivo recording techniques reveal that most areas of the brain outside of the SCN exhibit circadian rhythms in electrical activity in phase with locomotor activity and $180^{\circ}$ out of phase with electrical activity in the SCN. Evidence for weakly expressed molecular and electrical rhythmicity in extraSCN brain regions suggests that the SCN may play the role of "master synchronizer" rather than as the singular circadian oscillator controlling circadian behaviors. This model would fit with the results showing that SCN-ablated animals can show a "damped" circadian rhythm of locomotor activity after entrainment to food availability. This food-entrainable oscillator is likely in the brain but has not been located (Davidson et al., 2001). 
There have been hints that extra-SCN brain oscillators play a modifying role in circadian behaviors. For example, removal of the olfactory bulbs affects the rate of reentrainment in male rodents (Goel et al., 1998). Aschoff's studies of humans in bunkers, in which he observed that various autonomic and performance rhythms exhibited different ranges of entrainment (Aschoff and Wever, 1981), led him to the concept of internal temporal order and to the conclusion that the human circadian system was a hierarchically organized system of multiple oscillators. Although one need not have multiple circadian oscillators to obtain the appropriate timing of physiological processes (e.g., a single time base in a computer can provide output signals at multiple phases), there are theoretical advantages of multiple oscillators in the control and stability of physiological systems (Oatley and Goodwin, 1971).

It is clear that many aspects of circadian timing will depend on the establishment of appropriate and stable phase relationships among many central and peripheral oscillators. It will be important to understand how these relationships are established and how these non-SCN oscillators affect physiology and behavior.

\section{REFERENCES}

Albrecht U, Sun ZS, Eichele G, Lee CC (1997) A differential response of two putative mammalian circadian regulators, mper1 and mper2, to light. Cell 91:1055-1064.

Aschoff J, Wever RA (1976) Human circadian rhythms: a multioscillator system. Fed Proc 35:2326-2332.

Aschoff J, Wever RA (1981) The circadian system of man. In: Handbook of behavioral neurology, pp 311-331. New York: Plenum.

Bae K, Jin X, Maywood ES, Hastings MH, Reppert SM, Weaver DR (2001) Differential functions of mPer1, mPer2, and mPer3 in the SCN circadian clock. Neuron 30:525-536.

Balsalobre A, Damiola F, Schibler U (1998) A serum shock induces circadian gene expression in mammalian tissue culture cells. Cell 93:929-937.

Balsalobre A, Brown SA, Marcacci L, Tronche F, Kellendonk C, Reichardt HM, Schutz G, Schibler U' (2000) Resetting of circadian time in peripheral tissues by glucocorticoid signaling. Science 289:2344-2347.

Davidson AJ, Cappendijk SL, Stephan FK (2000) Feeding-entrained circadian rhythms are attenuated by lesions of the parabrachial region in rats. Am J Physiol Regul Integr Comp Physiol 278:R1296-R1304.

Davidson AJ, Aragona BJ, Werner RM, Schroeder E, Smith JC, Stephan FK (2001) Food-anticipatory activity persists after olfactory bulb ablation in the rat. Physiol Behav 72:231-235.

Geusz ME, Fletcher C, Block GD, Straume M, Copeland NG, Jenkins NA, Kay SA, Day RN (1997) Long-term monitoring of circadian rhythms in c-fos gene expression from suprachiasmatic nucleus cultures. Curr Biol 7:758-766.

Goel N, Lee TM, Pieper DR (1998) Removal of the olfactory bulbs delays photic reentrainment of circadian activity rhythms and modifies the reproductive axis in male Octodon degus. Brain Res 792:229-236.

Herzog ED, Takahashi JS, Block GD (1998) Clock controls circadian period in isolated suprachiasmatic nucleus neurons. Nat Neurosci 1:708-713.

Hida A, Koike N, Hirose M, Hattori M, Sakaki Y, Tei H (2000) The human and mouse Period1 genes: five well-conserved E-boxes additively contribute to the enhancement of mPer1 transcription. Genomics 65:224-233.

Inouye ST (1983) Does the ventromedial hypothalamic nucleus contain a self-sustained circadian oscillator associated with periodic feedings? Brain Res 279:53-63.
Inouye ST, Kawamura H (1979) Persistence of circadian rhythmicity in a mammalian hypothalamic "island" containing the suprachiasmatic nucleus. Proc Natl Acad Sci USA 76:5962-5966.

Inouye ST, Kawamura H (1982) Characteristics of a circadian pacemaker in the suprachiasmatic nucleus. J Comp Physiol [A] 146:153-160.

Klein DC, Moore RY, Reppert SM (1991) Suprachiasmatic nucleus: the mind's clock. New York: Oxford UP.

Kubota A, Inouye ST, Kawamura H (1981) Reversal of multiunit activity within and outside the suprachiasmatic nucleus in the rat. Neurosci Lett 27:303-308.

Liu C, Weaver DR, Strogatz SH, Reppert SM (1997) Cellular construction of a circadian clock: period determination in the suprachiasmatic nuclei. Cell 91:855-860.

Matsumoto S, Basil J, Jetton AE, Lehman MN, Bittman EL (1996) Regulation of the phase and period of circadian rhythms restored by suprachiasmatic transplants. J Biol Rhythms 11:145-162.

Meerlo P, Van den Hoofdakker RH, Koolhaas JM, Daan S (1997) Stress-induced changes in circadian rhythms of body temperature and activity in rats are not caused by pacemaker changes. J Biol Rhythms 12:80-92.

Meijer JH, Watanabe K, Schaap J, Albus H, Detari L (1998) Light responsiveness of the suprachiasmatic nucleus: long-term multiunit and single-unit recordings in freely moving rats. J Neurosci 18:9078-9087.

Oatley K, Goodwin BC (1971) The explanation and investigation of biological rhythms. In: Biological rhythms and human performance (Colquhoun WP, ed), pp 1-38. New York: Academic.

Paxinos G, Watson C (1998) The rat brain in stereotaxic coordinates. New York: Academic.

Ralph MR, Foster RG, Davis FC, Menaker M (1990) Transplanted suprachiasmatic nucleus determines circadian period. Science 247:975-978.

Rutter J, Reick M, Wu LC, McKnight SL (2001) Regulation of clock and NPAS2 DNA binding by the redox state of NAD cofactors. Science 293:510-514.

Shearman LP, Zylka MJ, Weaver DR, Kolakowski LF, Reppert SM (1997) Two period homologs: circadian expression and photic regulation in the suprachiasmatic nuclei. Neuron 19:1261-1269.

Stokkan KA, Yamazaki S, Tei H, Sakaki Y, Menaker M (2001) Entrainment of the circadian clock in the liver by feeding. Science 291:490-493.

Sun ZS, Albrecht U, Zhuchenko O, Bailey J, Eichele G, Lee CC (1997) RIGUI, a putative mammalian ortholog of the Drosophila period gene. Cell 90:1003-1011.

Tei H, Okamura H, Shigeyoshi Y, Fukuhara C, Ozawa R, Hirose M, Sakaki Y (1997) Circadian oscillation of a mammalian homologue of the Drosophila period gene. Nature 389:512-516.

Tosini G, Menaker M (1996) Circadian rhythms in cultured mammalian retina. Science 272:419-421.

Welsh DK, Logothetis DE, Meister M, Reppert SM (1995) Individual neurons dissociated from rat suprachiasmatic nucleus express independently phased circadian firing rhythms. Neuron 14:697-706.

Yamamoto S, Shigeyoshi Y, Ishida Y, Fukuyama T, Yamaguchi S, Yagita K, Moriya T, Shibata S, Takashima N, Okamura H (2001) Expression of the Per1 gene in the hamster: brain atlas and circadian characteristics in the suprachiasmatic nucleus. J Comp Neurol 430:518-532.

Yamazaki S, Kerbeshian MC, Hocker CG, Block GD, Menaker M (1998) Rhythmic properties of the hamster suprachiasmatic nucleus in vivo. J Neurosci 18:10709-10723.

Yamazaki S, Numano R, Abe M, Hida A, Takahashi R, Ueda M, Block GD, Sakaki Y, Menaker M, Tei H (2000) Resetting central and peripheral circadian oscillators in transgenic rats. Science 288:682-685.

Yan L, Takekida S, Shigeyoshi Y, Okamura H (1999) Per1 and Per2 gene expression in the rat suprachiasmatic nucleus: circadian profile and the compartment-specific response to light. Neuroscience 94:141-150.

Zheng B, Albrecht U, Kaasik K, Sage M, Lu W, Vaishnav S, Li Q, Sun ZS, Eichele G, Bradley A, Lee CC (2001) Nonredundant roles of the mPer1 and mPer2 genes in the mammalian circadian clock. Cell 105: 683-694. 